PROCEEDINGS OF THE

AMERICAN MATHEMATICAL SOCIETY

Volume 135, Number 9, September 2007, Pages 2713-2722

S 0002-9939(07)08829-6

Article electronically published on May 4, 2007

\title{
LEVEL ALGEBRAS WITH BAD PROPERTIES
}

\author{
MATS BOIJ AND FABRIZIO ZANELLO \\ (Communicated by Bernd Ulrich)
}

\begin{abstract}
This paper can be seen as a continuation of the works contained in the recent article (J. Alg., 305 (2006), 949-956) of the second author, and those of Juan Migliore (math. AC/0508067). Our results are:

1). There exist codimension three artinian level algebras of type two which do not enjoy the Weak Lefschetz Property (WLP). In fact, for $e \gg 0$, we will construct a codimension three, type two $h$-vector of socle degree $e$ such that all the level algebras with that $h$-vector do not have the WLP. We will also describe the family of those algebras and compute its dimension, for each $e \gg 0$.

2). There exist reduced level sets of points in $\mathbf{P}^{3}$ of type two whose artinian reductions all fail to have the WLP. Indeed, the examples constructed here have the same $h$-vectors we mentioned in 1$)$.

3). For any integer $r \geq 3$, there exist non-unimodal monomial artinian level algebras of codimension $r$. As an immediate consequence of this result, we obtain another proof of the fact (first shown by Migliore in the abovementioned preprint, Theorem 4.3) that, for any $r \geq 3$, there exist reduced level sets of points in $\mathbf{P}^{r}$ whose artinian reductions are non-unimodal.
\end{abstract}

\section{INTRODUCTION}

The study of the properties of level algebras, both in the artinian and in the higher-dimensional case, is an intriguing issue in commutative algebra, not only because of the intrinsic importance of these algebras, but also due to the relevance of their applications to several other areas of mathematics, such as algebraic geometry, invariant theory, combinatorics, etc. There is an excellent broad overview of level algebras in the memoir [GHMS, and we refer to it for the most comprehensive bibliography up to the year 2003. However, although a remarkable amount of research on level algebras has been performed over the last few years, several interesting problems are still open. The purpose of this note is to provide the solution to three of those problems.

We will first focus on the Weak Lefschetz Property (WLP, in brief; see below for the definition), which is a fundamental and very natural property of artinian algebras, and has recently received much attention (e.g., see HMNW and MM and their bibliographies). In codimension $r \geq 4$, it is known that the WLP may

Received by the editors December 15, 2005 and, in revised form, May 20, 2006.

2000 Mathematics Subject Classification. Primary 13H10; Secondary 13D40, 13E10, 14 M05.

Key words and phrases. Type 2 level algebra, Weak Lefschetz Property, monomial algebra, non-unimodality.

The second author is funded by the Göran Gustafsson Foundation.

(C)2007 American Mathematical Society Reverts to public domain 28 years from publication 
fail even for Gorenstein algebras (see Ikeda [k Example 4.4], and the first author [Bo2, Theorem 3.6], for $r=4$; see also [St, Example 4.3], BI], BL and [Bo1, which supply examples of Gorenstein algebras of codimension $r \geq 5$ that are nonunimodal, and therefore, a fortiori, without the WLP).

The second author (see [Za, Corollary 4 and Proposition 8]) has recently shown that there even exist codimension 3 artinian level algebras which do not enjoy the WLP, but could only provide examples having type greater than or equal to 3 . (The idea, at least for type three, was to use an inverse system generated by three forms of the same degree, two suitably chosen in two variables, $y_{1}, y_{2}$, and the third being generic in variables $y_{2}, y_{3}$.) Therefore, only the problem as to whether there exist Gorenstein or type 2 level algebras of codimension 3 without the WLP remained open. As for the Gorenstein case, so far the most important result is HMNW, Corollary 2.4], which proves, with a beautiful geometric argument, that all complete intersections of codimension 3 enjoy the WLP.

The first result of this paper is the solution to the type 2 case: indeed, we will show that, for any $e \gg 0$, there exist codimension 3, type 2 level algebras of socle degree $e$ which do not enjoy the WLP. Our examples are also surprisingly simple. In fact, the inverse system modules we will construct are generated by one monomial and one binomial. Actually, we will prove more: we will determine the $h$-vectors $h^{(e)}$ of the level algebras without the WLP mentioned above and show that, in fact, all the level algebras having those $h$-vectors do not enjoy the WLP. For each $h^{(e)}$, we will also completely describe the family of level algebras with $h$-vector $h^{(e)}$ and show that it is an irreducible variety of dimension $3(e+1)$.

The recent preprint of Migliore, $\mathrm{Mi}$, has extended some of the results of [Za] to (one-dimensional) level algebras of type $t \geq 3$ of reduced sets of points of the projective space $\mathbf{P}^{3}$. The second result of this note, obtained by employing a technique rather similar to that of Migliore, is the construction of type 2, reduced level sets of points of $\mathbf{P}^{3}$ whose artinian reductions all fail to have the WLP. Indeed, these examples have the same $h$-vectors as those supplied in our first result mentioned above. Thus, as far as the WLP is concerned, both for artinian and for one-dimensional codimension 3 level algebras the non-existence problem now remains open only in the Gorenstein case.

In $\mathrm{Za}$, Theorem 3], the second author has also proved the existence of codimension 3 artinian level algebras having a non-unimodal $h$-vector. (These examples all have a relatively large type, and for small types the unimodality problem is still open.) However, none of his algebras were monomial, and the problem as to whether there exist non-unimodal monomial level algebras (of any codimension) remained unsolved.

The third main result of this note is the solution to that problem: we will show that, for every $r \geq 3$, there exist codimension $r$ monomial artinian level algebras having a non-unimodal $h$-vector. As an immediate corollary, since monomial artinian ideals lift to ideals of points, we have another proof of the existence of reduced level sets of points in $\mathbf{P}^{r}$ (for every $r \geq 3$ ) whose artinian reductions are non-unimodal (this result was first shown in [Mi, Theorem 4.3]).

\section{Setup}

Let us now introduce the main definitions we will need in this note. We consider standard graded algebras $A=R / I$, where $R=k\left[x_{1}, \ldots, x_{r}\right], I$ is a homogeneous 
ideal of $R, k$ is a field of characteristic zero and the $x_{i}$ 's all have degree 1. Our algebras will be either artinian or Cohen-Macaulay (CM) (mostly of dimension $d=1$, as in the case of points in the projective space).

When $A$ is artinian, the $h$-vector of $A$ is $h(A)=h=\left(h_{0}, h_{1}, \ldots, h_{e}\right)$, where $h_{i}=\operatorname{dim}_{k} A_{i}$ and $e$ is the last index such that $\operatorname{dim}_{k} A_{e}>0$. Since we may suppose, without loss of generality, that $I$ does not contain non-zero forms of degree 1 , the codimension of $A$ is $r=h_{1}$. In general, when $A$ is CM of dimension $d$, its $h$-vector is that of any of its artinian reductions (i.e., artinian quotients of $A$ by a regular sequence), and its codimension is $r-d$.

In the artinian case, the socle of $A$ is the annihilator of the maximal homogeneous ideal $\bar{m}=\left(\overline{x_{1}}, \ldots, \overline{x_{r}}\right) \subseteq A$, namely $\operatorname{soc}(A)=\{a \in A \mid a \bar{m}=0\}$. Since $\operatorname{soc}(A)$ is a homogeneous ideal, we define the socle vector of $A$ as $s(A)=s=\left(s_{0}, s_{1}, \ldots, s_{e}\right)$, where $s_{i}=\operatorname{dim}_{k} \operatorname{soc}(A)_{i}$. Note that $h_{0}=1, s_{0}=0$ and $s_{e}=h_{e}>0$. The integer $e$ is called the socle degree of $A$ (or of $h$ ). The type of the socle vector $s$ (or of the algebra $A)$ is type $(s)=\sum_{i=0}^{e} s_{i}$.

If $s=\left(0,0, \ldots, 0, s_{e}=t\right.$ ), we say that the graded algebra $A$ is level (of type $t$ ). If, moreover, $t=1$, then $A$ is Gorenstein.

Since the graded Betti numbers of a minimal free resolution (MFR) of a CM algebra $A$ are preserved when we consider the MFR of any artinian reduction $A^{\prime}$ of $A$, and since the non-zero entries of the socle vector of $A^{\prime}$ are given by the graded Betti numbers of the last module of its MFR, then we can define a CM algebra as level of type $t$ when its artinian reductions are level of type $t$.

An artinian algebra $A=\bigoplus_{i=0}^{e} A_{i}$ is said to have the Weak Lefschetz Property $(W L P)$ if there exists a linear form $l \in R$ such that, for all indices $i=0,1, \ldots, e-1$, the multiplication map " $l$ " between the $k$-vector spaces $A_{i}$ and $A_{i+1}$ has maximal rank.

Let us now recall the main facts of the theory of inverse systems, or Macaulay duality, which will be a fundamental tool in this note. For a complete introduction, we refer the reader to $\mathrm{Ge}$ and [IK].

Let $S=k\left[y_{1}, \ldots, y_{r}\right]$, and consider $S$ as a graded $R$-module where the action of $x_{i}$ on $S$ is partial differentiation with respect to $y_{i}$.

There is a one-to-one correspondence between graded artinian algebras $R / I$ and finitely generated graded $R$-submodules $M$ of $S$, where $I=A n n(M)$ is the annihilator of $M$ in $R$ and, conversely, $M=I^{-1}$ is the $R$-submodule of $S$ which is annihilated by $I$ (cf. [Ge, Remark 1], p. 17).

If $R / I$ has socle vector $s$, then $M$ is minimally generated by $s_{i}$ elements of degree $i$, for $i=1,2, \ldots, e$, and the $h$-vector of $R / I$ is given by the number of linearly independent partial derivatives obtained in each degree by differentiating the generators of $M$ (cf. Ge, Remark 2], p. 17).

In particular, artinian level algebras of type $t$ and socle degree $e$ correspond to $R$-submodules of $S$ minimally generated by $t$ elements of degree $e$.

It is easy to see that the inverse system module $M$ of an ideal $I$ is generated by monomials if and only if $I$ is a monomial ideal (i.e., it is also generated by monomials). 


\section{Non-WLP FOR TYPE TWO-ThE ARTINIAN CASE}

We now turn our attention to the first result of this note: as we have mentioned above, for each $e \gg 0$, we will construct a codimension $3 h$-vector of socle degree $e$, such that all the type 2 level algebras having this $h$-vector do not enjoy the WLP.

Let $R=k\left[x_{1}, x_{2}, x_{3}\right], S=k\left[y_{1}, y_{2}, y_{3}\right]$, and let "०" indicate the operation of differentiation on inverse system modules.

Definition 3.1. For every integer $e$, define the family $\mathcal{F}_{e}$ by

$$
\mathcal{F}_{e}=\{R / \operatorname{Ann}(M) \mid M=(F, G) \subset S, \text { where } \operatorname{deg} F=\operatorname{deg} G=e \text { and }
$$

$l_{1} \circ F=\left(l_{1} l_{2}\right) \circ G=0$ for some, not necessarily distinct, linear forms $\left.l_{1}, l_{2} \in R\right\}$,

and define the $h$-vector $h^{(e)}=\left(h_{0}, h_{1}, \ldots, h_{e}\right)$ by

$$
h_{d}=\min \{2 d+1, e+2,3(e-d)+2\},
$$

for $d=0,1, \ldots, e$.

Theorem 3.2. The generic element of $\mathcal{F}_{e}$ has $h$-vector $h^{(e)}$, and for $e \geq 6$ all level algebras with $h$-vector $h^{(e)}$ are in $\mathcal{F}_{e}$, which is an irreducible variety of dimension $3(e+1)$.

Moreover, for odd $e \geq 9$ and even $e \geq 12$, any level algebra with $h$-vector $h^{(e)}$ does not satisfy the Weak Lefschetz Property.

Proof. We start by finding an element in $\mathcal{F}_{e}$ with $h$-vector $h^{(e)}$. Namely, we want to show that

$$
M=\left\langle y_{1}^{[2 e / 3]} y_{3}^{[e / 3]}, y_{2}^{\lceil e / 2\rceil} y_{3}^{\lfloor e / 2\rfloor}+y_{1}^{[e / 3]} y_{3}^{[2 e / 3]}\right\rangle \subset S
$$

is the inverse system module associated to a type 2 level algebra $A$, which is clearly in $\mathcal{F}_{e}$, whose $h$-vector is $h^{(e)}$. Recall that $\lceil a\rceil$ indicates the smallest integer greater than or equal to $a,\lfloor a\rfloor$ the largest integer less that or equal to $a$, and $[a]$ the integer nearest to $a$, assuming that this can be uniquely determined. There are six different cases to consider, one for each equivalence class of $e$ modulo 6 . We will only prove the result for $e \equiv 4(\bmod 6)$; the arguments for the other cases are entirely similar and therefore will be omitted.

Hence let $e=6 n+4$. Thus,

$$
M=(F, G)=\left(y_{1}^{4 n+3} y_{3}^{2 n+1}, y_{2}^{3 n+2} y_{3}^{3 n+2}+y_{1}^{2 n+1} y_{3}^{4 n+3}\right),
$$

and we want to show that the $h$-vector of $R / A n n(M)$ is $(1,3,5, \ldots, 6 n+5,6 n+$ $6,6 n+6, \ldots, 6 n+6,6 n+5,6 n+2, \ldots, 11,8,5,2)$, where the first $6 n+5$ is in degree $3 n+2$, and $6 n+6$ appears $n$ times (from degree $3 n+3$ to degree $4 n+2$ ).

It is easy to see that, for each $i=1,2, \ldots, 2 n+1$, the $i$-th (linearly independent partial) derivatives of $F$ are $i+1$ (i.e., they are as many as they can possibly be for a form in two variables), and the derivatives of $G$ are $2 i+1$ (since $y_{1} y_{2}$ does not appear in the summands of $G$ ). Furthermore, the first $2 n+1$ derivatives of $F$ and $G$ are clearly disjoint. Hence, for $1 \leq i \leq 2 n+1, h_{e-i}=(i+1)+(2 i+1)=3 i+2$. This proves that $h$ ends with $(\ldots, 6 n+5,6 n+2, \ldots, 8,5,2)$ from degree $4 n+3$ on.

It is immediate to see that $h_{1}=3$ and $h_{2}=5$ (since $x_{1} x_{2}$ is the only degree 2 monomial annihilating both $F$ and $G$ ). Hence, by Macaulay's theorem and the properties of the binomial expansion, it is easy to see that the equality $h_{d}=2 d+1$ for $d \leq 3 n+2$ follows once we show that $h_{3 n+3}=6 n+6$. Therefore, it remains to prove that $h_{3 n+3}=h_{3 n+4}=\ldots=h_{4 n+2}=6 n+6$. 
A simple computation shows that $\operatorname{Ann}(M)_{4 n+2}$ is generated (as a $k$-vector space) by monomials, with $A_{4 n+2}$ spanned by the classes of the following two sets of monomials:

$$
T_{1}=\left\{x_{1}^{4 n+2}, x_{1}^{4 n+1} x_{3}, \ldots, x_{3}^{4 n+2}\right\}
$$

(i.e., all the $4 n+3$ degree $4 n+2$ monomials of $R$ in variables $x_{1}$ and $x_{3}$ only), and

$$
T_{2}=\left\{x_{2}^{3 n+2} x_{3}^{n}, x_{2}^{3 n+1} x_{3}^{n+1}, \ldots, x_{2}^{n} x_{3}^{3 n+2}\right\},
$$

which has cardinality $3 n+2-n+1=2 n+3$. Thus, $h_{4 n+2}=(4 n+3)+(2 n+3)=$ $6 n+6$, as desired.

Now, for each $i=1,2, \ldots, n-1$, the monomials generating $A_{4 n+2-i}$ are of course obtained by differentiating the monomials of $T_{1}$ and $T_{2}$. Those coming from $T_{1}$ are exactly the $4 n+3-i$ monomials of degree $4 n+2-i$ in variables $x_{1}$ and $x_{3}$ only, while those obtained from $T_{3}$ are $x_{2}^{3 n+2} x_{3}^{n-i}, x_{2}^{3 n+1} x_{3}^{n-i+1}, \ldots, x_{2}^{n-i} x_{3}^{3 n+2}$, which are $2 n+3+i$. Hence, for $i=1,2, \ldots, n-1, h_{4 n+2-i}=(4 n+3-i)+(2 n+3+i)=6 n+6$, as we wanted to show.

We now proceed to prove that $h^{(e)}$ is an upper bound for the $h$-vector of any member of the family $\mathcal{F}_{e}$. For this purpose, let $A=R / I$ be an element of $\mathcal{F}_{e}$, where $I=\operatorname{Ann}(F, G)$ for some $F$ and $G$ in $S_{e}$.

Since $l_{1} l_{2} \circ F=l_{1} l_{2} \circ G=0$, for some linear forms $l_{1}$ and $l_{2}$, we have that $l_{1} l_{2} \in I$. Thus the $h$-vector of $A$ is bounded from above by the Hilbert function of $R /\left(l_{1} l_{2}\right)$, i.e., $2 d+1$.

The inverse system module $I^{-1}$ is generated by $F$ and $G$ with the two relations $l_{1} \circ F=0$ and $l_{1} l_{2} \circ G=0$. Thus we get the inequality

$$
h(A)_{e-d} \leq(d+1)+(2 d+1)=3 d+2, \quad \text { for } d=0,1, \ldots, e .
$$

We now have to establish the inequality $h(A)_{d} \leq e+2$. By a change of variables we may assume that $l_{1}=x_{1}$. The derivatives of $F$ and $l_{2} \circ G$ with respect to $y_{1}$ are all zero. This means that the derivatives of degree $e-d$ of $F$ and $l_{2} \circ G$ live in a space of dimension $e-d+1$. What remains are the derivatives of $G$ obtained by operating with forms of degree $d$ in $x_{2}$ and $x_{3}$, and the dimension of their span is bounded from above by $d+1$. Thus, in total, the $h$-vector of $A$ in degree $e-d$ is bounded from above by $(e-d+1)+(d+1)=e+2$.

We now prove that any level algebra with $h$-vector $h^{(e)}$, for $e \geq 6$, is in the family $\mathcal{F}_{e}$. Let $A=R / I$ be any artinian level algebra with $h(A)=h^{(e)}$. By the value of the $h$-vector in degree two, we see that there is a quadratic form $q$ in the ideal $I$. Let $F$ and $G$ be the generators of the inverse system module $I^{-1}$. By the value of the $h$-vector in degree $e-1$, we have a linear relation among $F$ and $G$, i.e., there are linear forms $l_{1}$ and $l_{2}$ such that $l_{1} \circ F=l_{2} \circ G$. Suppose that both of these forms are non-zero and linearly independent. Then we can find a form $H$ such that $F=l_{2} \circ H$ and $G=l_{1} \circ H$. Since $e \geq 6$, we have that $h(A)_{e-2}=8$ and we need eight linearly independent second derivatives of $F$ and $G$. There are at most nine possible such derivatives, since $F$ and $G$ are derivatives of $H$, and in addition we have $q \circ F=q \circ G=0$. Thus $h(A)_{e-2} \leq 7$, and we can conclude that the linear relation can be written as $l_{1} \circ F=0$. Hence we have that $x_{1} l_{1} \circ F=x_{2} l_{1} \circ F=x_{3} l_{1} \circ F=0$. Together with $q \circ F=q \circ G=0$, we get five quadratic relations among $F$ and $G$. Because of the $h$-vector, these relations must be linearly dependent, i.e., $q=l_{1}\left(a x_{1}+b x_{2}+c x_{3}\right)=l_{1} l_{2}$ for some linear form $l_{2}=a x_{1}+b x_{2}+c x_{3}$. This proves that $A$ is in the family $\mathcal{F}_{e}$. 
Now, we can show that any element of $\mathcal{F}_{e}$ with Hilbert function $h^{(e)}$ does not satisfy the WLP for odd $e \geq 9$ and even $e \geq 12$.

Let $l$ be a generic linear form in $A$. Then the ideal $(l)$ is a cyclic submodule of $A$ and hence isomorphic to a quotient $B=A /(0: l)=R /(I: l)$. Since $(I: l)^{-1}=$ $(l \circ F, l \circ G)$, we have that $B$ is an element of $\mathcal{F}_{e-1}$. Hence all entries of the $h$-vector of $B$ are bounded by $(e-1)+2=e+1$. This means that the Hilbert function of $(0: l)$ is at least 1 in degrees where $h(A)_{d}=e+2$. Thus the multiplication by $l$ fails to be injective in these degrees. For odd $e \geq 9$ and even $e \geq 12$, we have that $h^{(e)}$ attains the value $e+2$ at least twice. Thus, in these cases, the algebra $A$ does not have the WLP.

We can prove that the family $\mathcal{F}_{e}$ is irreducible of dimension $3(e+1)$ in the following way. We map the family onto the space of reducible conics, $q=l_{1} l_{2}$, which has dimension four. The fibers are irreducible of dimension $(e+1)+(2 e+1)-3=$ $3 e-1$, since the choice of $F$ and $G$ corresponds to $e+1$ and $2 e+1$, respectively, but $(F, G)=(a F, b G+c F)$, whenever $a$ and $b$ are non-zero. Thus the family $\mathcal{F}_{e}$ is irreducible of dimension $4+(3 e-1)=3(e+1)$, as we wanted to show.

\section{NON-WLP FOR TYPE TWO-The CASE OF POINTS}

We will now show that our first result can be "lifted" to homogeneous coordinate rings of points of the projective space $\mathbf{P}^{3}$ which are level of type two and whose artinian reductions all fail to satisfy the WLP. The idea is rather similar to that of Migliore ( $\mathrm{Mi}]$ ), which involved the use of sets of points on two different hyperplanes. In order to obtain the desired $h$-vector and type we need to be careful in the choice of the points on the hyperplanes. We can view the technique we will employ here as a basic double linkage, as in Migliore's work, although we will not use this description in the proof.

For any integer $e$, define the positive integers $c_{0}, c_{1}, c_{2}$ and $q_{0}, q_{1}, q_{2}, q_{3}$ such that: $c_{0} \leq c_{1} \leq c_{2} \leq c_{0}+1, c_{0}+c_{1}+c_{2}=e,\left|q_{i}-q_{j}\right| \leq 1$ for all $i$ and $j$, and

$$
\left\{\begin{array}{l}
q_{0}+q_{1}=c_{0}+1 \\
q_{1}+q_{2}=c_{1}+1 \\
q_{2}+q_{3}=c_{2}+1
\end{array}\right.
$$

Equivalently, in a more compact fashion, we can write

$$
c_{i}=\left\lfloor\frac{e+i}{3}\right\rfloor, \quad \text { for } i=0,1,2,
$$

and

$$
q_{i}=\left\lfloor\frac{e+i-1}{2}\right\rfloor-\left\lfloor\frac{e+i-2}{3}\right\rfloor, \quad \text { for } i=0,1,2,3 .
$$

Define the ideal $I \subset R=k\left[x_{0}, x_{1}, x_{2}, x_{3}\right]$ by

$$
\begin{aligned}
I= & \left(l_{0} l_{1}, l_{0}\left(C_{0} Q_{2}-C_{1} Q_{0}\right), l_{0}\left(C_{1} Q_{3}-C_{2} Q_{1}\right),\right. \\
& \left.C_{0} Q_{1} Q_{2}-C_{1} Q_{0} Q_{1}, C_{1} Q_{2} Q_{3}-C_{2} Q_{1} Q_{2}, C_{0} Q_{2} Q_{3}-C_{2} Q_{0} Q_{1}\right),
\end{aligned}
$$

where $l_{0}, l_{1}$ are linear forms and $C_{0}, C_{1}, C_{2}, Q_{0}, Q_{1}, Q_{2}, Q_{3}$ are forms of degrees $c_{0}, c_{1}, c_{2}, q_{0}, q_{1}, q_{2}, q_{3}$.

Theorem 4.1. For a generic choice of the forms $l_{0}, l_{1}, C_{0}, C_{1}, C_{2}, Q_{0}, Q_{1}, Q_{2}$ and $Q_{3}$, the ideal $I \subset R$ defined above is the ideal of a reduced level set of points of $\mathbf{P}^{3}$ of type two, having h-vector $\left(1, h_{1}, \ldots, h_{e}\right)$, where

$$
h_{d}=\min \{2 d+1, e+2,3(e-d)+2\}, \quad \text { for } d=0,1, \ldots, e .
$$


By Theorem 3.2, no artinian reduction of $A=R / I$ satisfies the $W L P$.

Proof. Let us consider the ideal $I_{1}$ of $R$, generated by the maximal minors of the matrix

$$
\left(\begin{array}{ccc}
C_{0} & C_{1} & C_{2} \\
Q_{0} Q_{1} & Q_{1} Q_{2} & Q_{2} Q_{3}
\end{array}\right)
$$

and the ideal $(f, g)$, where $f=C_{1} Q_{3}-C_{2} Q_{1}$ and $g=C_{0} Q_{2}-C_{1} Q_{0}$. The ideal $I_{1}$ is the ideal of a curve containing the complete intersection curve defined by $(f, g)$. The residual scheme is given by

$$
I_{1}:(f, g)=\left(Q_{0} Q_{1}, Q_{1} Q_{2}, Q_{2} Q_{3}\right)=\left(Q_{0}, Q_{2}\right) \cap\left(Q_{1}, Q_{2}\right) \cap\left(Q_{1}, Q_{3}\right),
$$

that is, the intersection of three complete intersection ideals.

For general forms $Q_{0}, Q_{1}, Q_{2}, Q_{3}$, each complete intersection ideal is radical, and so is their intersection $I_{1}:(f, g)$. The complete intersection ideal $(f, g)$ is also radical for a general choice of the above seven forms. To see this, we can choose a special set of forms and show that $(f, g)$ is radical for this choice. In order to do that, let $C_{1}=0$. Then $(f, g)=\left(C_{0} Q_{2}, C_{2} Q_{1}\right)=\left(C_{0}, C_{2}\right) \cap\left(C_{0}, Q_{1}\right) \cap\left(Q_{2}, C_{2}\right) \cap\left(Q_{1}, Q_{2}\right)$ is clearly radical if we take $C_{1}, C_{2}, Q_{1}, Q_{2}$ to be products of generic linear forms. Thus, since $I=\left(\left(l_{0}\right)+I_{1}\right) \cap\left(l_{1}, f, g\right)$, and a general linear section of a reduced curve is reduced, we have that $I$ is also radical.

In order to show that the algebra defined by the ideal $I$ is level, let us start with the polynomial ring in nine variables

$$
T=k\left[x, y, z_{0}, z_{1}, z_{2}, w_{0}, w_{1}, w_{2}, w_{3}\right],
$$

and consider the ideal $J \subseteq T$ defined by

$$
J=\left(x y, y f, y g, m_{1}, m_{2}, m_{3}\right),
$$

where $m_{1}, m_{2}, m_{3}$ are the maximal minors of

$$
\left(\begin{array}{ccc}
z_{0} & z_{1} & z_{2} \\
w_{0} w_{1} & w_{1} w_{2} & w_{2} w_{3}
\end{array}\right)
$$

and $f=m_{1} / w_{2}=z_{1} w_{3}-z_{2} w_{1}, g=m_{3} / w_{1}=z_{0} w_{2}-z_{1} w_{0}$.

A simple computation with Macaulay2 shows that the MFR of $J$ is:

$$
0 \rightarrow T^{2}(-6) \stackrel{\phi_{2}}{\longrightarrow} T^{6}(-4) \oplus T(-5) \stackrel{\phi_{1}}{\longrightarrow} T^{5}(-3) \oplus T(-2) \stackrel{\phi_{0}}{\longrightarrow} J \rightarrow 0,
$$

where the maps $\phi_{i}$ are given by the matrices:

$$
\begin{gathered}
\phi_{0}=\left(\begin{array}{ccccccc}
x y & y f & y g & m_{1} & m_{2} & m_{3}
\end{array}\right), \\
\phi_{1}=\left(\begin{array}{ccccccc}
0 & 0 & 0 & f & g & 0 & 0 \\
w_{2} & -w_{0} & 0 & -x & 0 & 0 & 0 \\
0 & -w_{3} & w_{1} & 0 & -x & 0 & 0 \\
-y & 0 & 0 & 0 & 0 & z_{0} & w_{0} w_{1} \\
0 & -y & 0 & 0 & 0 & z_{1} & w_{1} w_{2} \\
0 & 0 & -y & 0 & 0 & z_{2} & w_{2} w_{3}
\end{array}\right), \quad \phi_{2}=\left(\begin{array}{ccc}
w_{0} w_{1} & x z_{0} \\
w_{1} w_{2} & x z_{1} \\
w_{2} w_{3} & x z_{2} \\
0 & g \\
0 & -f \\
0 & x y \\
y & 0
\end{array}\right) .
\end{gathered}
$$

Thus, from the last module of the MFR, we deduce that the ideal $J$ is level of type two. Now we want to show that $I$ is obtained as a reduction of $J$ by a regular sequence.

Let $J^{\prime}$ be the extension of $J$ in the polynomial ring

$$
R \otimes_{k} T=k\left[x_{0}, x_{1}, x_{2}, x_{3}, x, y, z_{0}, z_{1}, z_{2}, w_{0}, w_{1}, w_{2}, w_{3}\right] .
$$


Hence we have that $I$ is obtained from $J^{\prime}$ by reduction using the sequence $\left(x-l_{0}, y-l_{1}, z_{0}-C_{0}, z_{1}-C_{1}, z_{2}-C_{2}, w_{0}-Q_{0}, w_{1}-Q_{1}, w_{2}-Q_{2}, w_{3}-Q_{3}\right)$.

In order to prove that this sequence is regular for a general choice of the forms, it suffices to show that the algebra $k\left[x_{0}, x_{1}, x_{2}, x_{3}\right] / I$ has dimension at most 1 . Indeed, we have that $I$ contains the regular sequence $\left(l_{0} l_{1}, Q_{2} f, Q_{1} g\right)$, as desired. Therefore, $R / I$ is the coordinate ring of a type two, level set of points of $\mathbf{P}^{3}$, since it is the reduction of a type two level algebra.

It now remains to check that the $h$-vector of $R / I$ is that of the statement. Notice that the algebra $A^{\prime}=R /\left(I, x_{3}\right)=k\left[x_{0}, x_{1}, x_{2}\right] / I^{\prime}$ is an artinian reduction of the algebra $R / I$ ( since $x_{3}$ is a non-zero divisor of $R / I$ ), and therefore the $h$-vector $h=\left(h_{0}, h_{1}, \ldots, h_{e}\right)$ and the graded Betti numbers $\beta_{i, j}$ are the same for the two algebras.

It is easy to check that the generators of $I$ (and hence those of $I^{\prime}$ ) have degrees

$$
\begin{gathered}
2,1+c_{0}+q_{2}=\left\lfloor\frac{e+3}{2}\right\rfloor, 1+c_{1}+q_{3}=\left\lfloor\frac{e+4}{2}\right\rfloor, c_{0}+q_{1}+q_{2}=\left\lfloor\frac{2 e+3}{3}\right\rfloor, \\
c_{0}+q_{2}+q_{3}=\left\lfloor\frac{2 e+4}{3}\right\rfloor, c_{1}+q_{2}+q_{3}=\left\lfloor\frac{2 e+5}{3}\right\rfloor .
\end{gathered}
$$

The MFR and the $h$-vector of $A^{\prime}$ are related by the following well-known formula (e.g., see [FL, p. 131, point (j)]):

$$
h(z)(1-z)^{3}=1+\sum_{i, j}(-1)^{i} \beta_{i, j} z^{j},
$$

where we set $h(z)=\sum_{i=0}^{e} h_{i} z^{i}$.

From the maps $\phi_{i}$ described above, we have that the r.h.s. of (4.1) is

$$
\begin{aligned}
1 & -z^{2}-z^{1+c_{0}+q_{2}}-z^{1+c_{1}+q_{3}}-z^{c_{0}+q_{1}+q_{2}}-z^{c_{0}+q_{2}+q_{3}}-z^{c_{1}+q_{2}+q_{3}} \\
& +z^{1+c_{0}+q_{1}+q_{2}}+z^{1+c_{0}+q_{2}+q_{3}}+z^{1+c_{1}+q_{2}+q_{3}}+z^{2+c_{0}+q_{2}}+z^{2+c_{1}+q_{3}} \\
& +z^{c_{0}+c_{1}+q_{2}+q_{3}}+z^{q_{0}+q_{1}+c_{1}+q_{2}+q_{3}}-2 z^{e+3} \\
& =1-z^{2}-z^{\lfloor(e+3) / 2\rfloor}-z^{\lfloor(e+4) / 2\rfloor}-z^{\lfloor(2 e+3) / 3\rfloor}-z^{\lfloor(2 e+4) / 3\rfloor} \\
& -z^{\lfloor(2 e+5) / 3\rfloor}+z^{\lfloor(2 e+6) / 3\rfloor}+z^{\lfloor(2 e+7) / 3\rfloor}+z^{\lfloor(2 e+8) / 3\rfloor} \\
& +z^{\lfloor(e+5) / 2\rfloor}+z^{\lfloor(e+6) / 2\rfloor}+z^{e+1}+z^{e+2}-2 z^{e+3} .
\end{aligned}
$$

A standard (but tedious) computation shows that the entries of the $h$-vector $h$ of the statement satisfy the equality (4.1), as we desired. This completes the proof of the theorem.

\section{Non-Unimodality FOR MONOMial LeVEL ALGEBRAS}

We will next show the third result of this note: namely, as we said in the introduction, we will prove the existence of non-unimodal monomial (artinian) level algebras of codimension $r$, for any $r \geq 3$.

Remember that the (degree) lexicographic order is a total ordering, briefly indicated by "> ", on the monomials of $S$ such that, if $P=y_{1}^{p_{1}} \cdots y_{r}^{p_{r}}$ and $Q=y_{1}^{q_{1}} \cdots y_{r}^{q_{r}}$ have the same degree, then $P>Q$ if and only if the first non-zero difference $p_{i}-q_{i}$ is positive.

Example 5.1. Let us consider the inverse system module $M \subset S=k\left[y_{1}, y_{2}, y_{3}\right]$ generated by the last (according to the lexicographic order) 36 monomials of degree 
12 , namely $M=\left\langle y_{1}^{2} y_{2}^{10}, y_{1}^{2} y_{2}^{9} y_{3}, \ldots, y_{3}^{12}\right\rangle$. An easy computation shows that the $h$ vector of $R / \operatorname{Ann}(M)$ is

$$
(1,3,6,9,12,15,18,21,24,27,30,33,36) .
$$

Notice now that the form $F=y_{1}^{6} y_{2}^{3} y_{3}^{3}$ has all of its partial derivatives of order 1 , 2 and 3 (which span vector spaces of dimension 3, 6 and 10, respectively) distinct from the partial derivatives of the forms generating $M$, since the latter forms and their derivatives are divisible by $y_{1}$ at most twice, whereas all the derivatives of order at most 3 of $F$ are divisible by $y_{1}$ at least thrice. Therefore, the inverse system module $M^{\prime}=\langle M, F\rangle$ generates an $h$-vector whose last four entries are:

$$
(27+10,30+6,33+3,36+1)=(37,36,36,37),
$$

whence the $h$-vector $h$ of the codimension three artinian monomial level algebra $R / A n n\left(M^{\prime}\right)$ is non-unimodal. In fact, we have

$$
h=(1,3,6,10,15,21,28,33,36,37,36,36,37) .
$$

Remark 5.2. i). The same construction can be used to produce infinitely many other examples. It suffices to generate $M$ by the last (according to the lexicographic order) $3 e$ monomial of degree $e$, and to consider the form $F=y_{1}^{e-6} y_{2}^{3} y_{3}^{3}$. Then $M^{\prime}=\langle M, F\rangle$ is, exactly as above, the inverse system module of a non-unimodal monomial level algebra of codimension 3 (its $h$-vector in fact ends with $(\ldots, 3 e+$ $1,3 e, 3 e, 3 e+1))$.

ii). The above examples can be extended to construct non-unimodal monomial level algebras of any codimension $r \geq 3$. Indeed, if a monomial level algebra $k\left[x_{1}, x_{2}, x_{3}\right] / \operatorname{Ann}(M)$ has $h$-vector $h=\left(1,3, h_{2}, \ldots, h_{e}\right)$, then the monomial level algebra $k\left[x_{1}, \ldots, x_{r}\right] / \operatorname{Ann}\left(\left\langle M, y_{4}^{e}, \ldots, y_{r}^{e}\right\rangle\right)$ has $h$-vector $h^{\prime}=\left(1, r, h_{2}+r-3, \ldots, h_{e}+\right.$ $r-3)$, which is clearly non-unimodal if $h$ is non-unimodal.

Hence we have shown:

Theorem 5.3. For every integer $r \geq 3$, there exist non-unimodal monomial artinian level algebras of codimension $r$.

Theorem 5.3 immediately extends to ideals of points of the projective space, since monomial artinian algebras lift to reduced sets of points. Hence, we have supplied another proof of the following recent result of Migliore ([Mi, Theorem 4.3]):

Corollary 5.4. For every integer $r \geq 3$, there exist reduced level sets of points in $\mathbf{P}^{r}$ whose artinian reductions are non-unimodal.

\section{Concluding questions}

We conclude our note with the following questions, which we regard as among the very next issues that now deserve to be addressed:

i) As far as the WLP is concerned, the existence problem for codimension 3 artinian level algebras, and for level sets of points of $\mathbf{P}^{3}$, now remains open only in the Gorenstein case: do all artinian Gorenstein algebras enjoy the WLP? (As we said in the introduction, HMNW, Corollary 2.4] provides a positive answer for the special case of complete intersections.) If not, are there also reduced sets of points of $\mathbf{P}^{3}$ whose artinian reductions are Gorenstein and do not enjoy the WLP? 
ii) As for monomial algebras, which is the largest type $t_{0}$ such that all the codimension 3 monomial level algebras of type $t \leq t_{0}$ are unimodal? It is easy to see that $t_{0}>0$, whereas here we have shown that $t_{0}$ is finite (in particular, $\left.t_{0} \leq 36\right)$.

iii) Similarly to what the second author has done in [Za, Remark 5, iii)] for arbitrary level algebras, can we extend Example 5.1 to $h$-vectors with three maxima? With any number of maxima?

\section{REFERENCES}

[BI] D. Bernstein and A. Iarrobino: A nonunimodal graded Gorenstein Artin algebra in codimension five, Comm. in Algebra 20 (1992), No. 8, 2323-2336. MR1172667 (93i:13012)

[Bo1] M. Boij: Graded Gorenstein Artin algebras whose Hilbert functions have a large number of valleys, Comm. in Algebra 23 (1995), No. 1, 97-103. MR1311776 (96h:13040)

[Bo2] M. Boij: Components of the space parameterizing graded Gorenstein Artinian algebras with a given Hilbert function, Pacific J. Math. 187 (1999), 1-11. MR.1674301 (2000j:14006)

[BL] M. Boij and D. Laksov: Nonunimodality of graded Gorenstein Artin algebras, Proc. Amer. Math. Soc. 120 (1994), 1083-1092. MR1227512 (94g:13008)

[FL] R. Fröberg and D. Laksov: Compressed Algebras, Conference on Complete Intersections in Acireale, Lecture Notes in Mathematics, No. 1092 (1984), 121-151, SpringerVerlag. MR0775880 (86f:13012)

[Ge] A.V. Geramita: Inverse Systems of Fat Points: Waring's Problem, Secant Varieties and Veronese Varieties and Parametric Spaces of Gorenstein Ideals, Queen's Papers in Pure and Applied Mathematics, No. 102, The Curves Seminar at Queen's (1996), Vol. X, 3-114. MR1381732 (97h:13012)

[GHMS] A.V. Geramita, T. Harima, J. Migliore and Y.S. Shin: The Hilbert Function of a Level Algebra, Memoirs of the Amer. Math. Soc., to appear.

[HMNW] T. Harima, J. Migliore, U. Nagel and J. Watanabe: The Weak and Strong Lefschetz Properties for Artinian K-Algebras, J. of Algebra 262 (2003), 99-126. MR.1970804 (2004b:13001)

[IK] A. Iarrobino and V. Kanev: Power Sums, Gorenstein Algebras, and Determinantal Loci, Springer Lecture Notes in Mathematics (1999), No. 1721, Springer, Heidelberg. MR 1735271 (2001d:14056)

[Ik] H. Ikeda: Results on Dilworth and Rees numbers of Artinian local rings, Japan. J. of Math. 22 (1996), 147-158. MR:1394376 (97g:13034)

[Macaulay2] D.R. Grayson and M.E. Stillman: Macaulay 2, a software system for research in algebraic geometry, available at http://www.math.uiuc.edu/Macaulay2/.

[Mi] J. Migliore: The geometry of the Weak Lefschetz Property, Canadian J. of Math., to appear (preprint: math.AC/0508067).

$[\mathrm{MM}] \quad$ J. Migliore and R. Miró-Roig: Ideals of general forms and the ubiquity of the Weak Lefschetz property, J. of Pure and Applied Algebra 182 (2003), 79-107. MR1978001 (2004c:13027)

[St] R. Stanley: Hilbert functions of graded algebras, Adv. Math. 28 (1978), 57-83. MR 0485835 (58:5637)

[Za] F. Zanello: A non-unimodal codimension 3 level h-vector, J. Alg. 305 (2006), 949956. MR2266862

Department of Mathematics, Royal Institute of Technology, S-100 44 Stockholm, SWEDEN

E-mail address: boij@math.kth.se

Department of Mathematics, Royal Institute of Technology, S-100 44 Stockholm, SWEDEN

E-mail address: zanello@math.kth.se 